\title{
CENTRAL RELATIONS ON LATTICES
}

\author{
DIETMAR SCHWEIGERT
}

(Received 2 February 1982)

Communicated by R. Lidl

\begin{abstract}
A maximal tolerance of a lattice $L$ without infinite chains is either a congruence or a central relation. A finite lattice $L$ is order-polynomially complete if and only if $L$ is simple and has no central relation. 1980 Mathematics subject classification (Amer. Math. Soc.): 06 B 10.
\end{abstract}

A binary relation $\rho$ is called compatible on a lattice $L$ if for $(a, b) \in \rho$ and $(c, d) \in \rho$ we have $(a \wedge c, b \wedge d) \in \rho$ and $(a \vee c, b \vee d) \in \rho$. A compatible binary relation $\rho$ on $L$ is a tolerance if $\rho$ is reflexive and symmetric. Of course every congruence relation is also a tolerance. First results on tolerances besides congruence relations were already derived in the papers of Hashimoto [5], Grätzer and E. T. Schmidt [7]. Chajda gives in [3] an overview of the theory. In this paper we study central relations which are tolerances having the property that there is a set $Z, \varnothing \subset Z \subset L$, such that $(z, a) \in \rho$ for every $a \in L$ if and only if $z \in Z$. $Z$ is called the center of $\rho$. The central relations play an important role in the theory of clones [8]. The aim of this paper is to show that every tolerance of a lattice $L$ without infinite chains which is not the all relation is either contained in a congruence or a central relation.

This result can be applied to characterize order-polynomially complete lattices. A lattice $L$ is called order-polynomially complete if every order-preserving function $f: L^{n} \rightarrow L$ is a polynomial function (= algebraic function). Lattices which correspond to finite projective geometries as well as the finite partition lattices have this combinatorial property. We show that a finite lattice is orderpolynomially complete if and only if $L$ is simple and has no central relation. This 
extends a result of Kindermann. Furthermore it is easy to check whether a finite lattice has a central relation because one has only to study single elements as a center for such a relation. This advantage can be used for deriving a new proof for a theorem of Wille [12]. In the following we call a binary relation $\rho$ non trivial if $\rho$ is neither the identity nor the all relation.

Proposition 1. Let $\rho$ be a nontrivial tolerance of a complete lattice $L$ and assume that $\rho$ is a complete sublattice of $L^{2}$. Define $a=\sup \{x \in L \mid(0, x) \in \rho\}$ and $b=\inf \{x \in L \mid(x, 1) \in \rho\}$. If $b \leqslant a$, then $\rho$ is a central relation.

Proof. Consider $Z=\{x \mid b \leqslant x \leqslant a\}$. Because of $(0, z)=(0, z) \wedge(z, z)$ we have $(0, z) \in \rho$ and similarly $(z, 1) \in \rho$. For every element $c \in L$ we have $(z, 1) \wedge(c, c)=(z \wedge c, c) \in \rho$ and $(z, 0) \in \rho$ hence $((z \wedge c) \vee z, c \vee 0)=(z, c)$ $\in \rho$. $Z$ is a proper subset of $L$ otherwise $\rho$ would be trivial.

Proposition 2. Let $\rho$ be an intransitive tolerance of the lattice $L$. Then there are $a, b, d \in L$ such that $(a, d) \in \rho,(d, d) \in \rho, a<d<b$ but $(a, b) \notin \rho$.

Proof. As $\rho$ is an intransitive tolerance we have $c, e, d \in L$ such that $(c, d) \in \rho$, $(d, e) \in \rho$ but $(c, e) \notin \rho$. If $d=0$ we have $(c, 0) \vee(0, e)=(c, e) \in \rho$. If $d=1$ we have $(c, 1) \wedge(1, e)=(c, e) \in \rho$. Therefore we have $0<d<1$. We put $a=c$ $\wedge e \wedge d$ and $b=c \vee e \vee d$. Then we have $(a, d) \in \rho$ and $(d, b) \in \rho$. If $(a, b) \in \rho$ then we have $(a, e) \in \rho$ and $(c, a) \in \rho$ hence $(c, e) \in \rho$. Therefore $(a, b) \notin \rho$.

LEMMA 3. Let $L$ have no infinite chains and let $\rho$ be an intransitive tolerance of $L$. If $\rho$ is not a central relation then there is a non trivial tolerance $\eta$ of $L$ such that $\rho \underset{\neq}{\subset} \eta$.

Proof. As $\rho$ is intransitive there is a triple $(a, d, b)$ such that $a<d<b$ and $(a, d) \in \rho,(d, b) \in \rho$ but $(a, b) \notin \rho$. We put $\eta=\langle\rho \cup\{(a, b),(b, a)\}\rangle$ the tolerance generated by $\rho$ and $(a, b),(b, a)$. Since $\rho^{\prime}=\rho \cup\{(a, b),(b, a)\}$ is reflexive and symmetric $\eta=\left\langle\rho^{\prime}\right\rangle$ is clearly the sublattice of $L^{2}$ generated by $\rho^{\prime}$. Assume $\eta$ is trivial, then we have $\eta=L^{2}$ and $(0,1) \in \eta$. Then there exists a term function $\varphi$ of $L^{2}$ with $\varphi\left(\left(c_{1}, e_{1}\right), \ldots,\left(c_{n}, e_{n}\right),(a, b),(b, a)\right)=(0,1)$ where $\rho \supset\left\{\left(c_{1}, e_{1}\right), \ldots,\left(c_{n}, e_{n}\right)\right\}[5]$ page 46. Since $\varphi$ is an isotonic function, we have $\varphi\left(\left(c_{1}, g_{1}\right), \ldots,\left(c_{n}, g_{n}\right),(a, b),(b, b)\right)=(0,1)$ where $g_{i}=e_{i} \vee c_{i}, i=1, \ldots, n$. We have that $\left(c_{i}, g_{i}\right) \in \rho$ and $c_{i} \leqslant g_{i}$. We split $\varphi=(\psi, \psi)$ in two term functions and we have $\psi\left(c_{1}, \ldots, c_{n}, a, b\right)=0, \psi\left(g_{1}, \ldots, g_{n}, b, b\right)=1$. Furthermore we put $F((x, y))=\varphi\left(\left(c_{1}, g_{1}\right), \ldots,\left(c_{n}, g_{n}\right),(x, y),(b, b)\right), f(x)=\psi\left(c_{1}, \ldots, c_{n}, x, b\right)$ and 
$g(x)=\psi\left(g_{1}, \ldots, g_{n}, x, b\right)$. We have $f(x) \leqslant g(x)$ for every $x \in L$. Consider $d_{*}=$ $\inf \{x \mid(x, d) \in \rho, x \in L\}$ and $d^{*}=\sup \{x \mid(x, d) \in \rho, x \in L\}$. Then we have $F\left(d_{*}, d\right)=(0, s) \in \rho$ since $\left(d_{*}, d\right) \in \rho$ and $(a, d) \in \rho$ implies $d_{*} \leqslant a$ and similarly $F\left(d, d^{*}\right)=(t, 1) \in \rho$ for $t \in L$. We have $f(d)=t \leqslant g(d)=s$. Hence we have $(0, s) \in \rho$ and $(s, s) \vee(t, 1)=(s, 1) \in \rho . s$ is an element of a center of $\rho$. Contradiction.

From this lemma it follows

THEOREM 4. A maximal tolerance of a lattice $L$ with no infinite chains is either a congruence relation or a central relation.

This theorem can be applied to the following result of M. Kindermann in [7]. A finite lattice is order-polynomially complete if and only if $L$ has only trivial tolerances. Therefore we have

THEOREM 5. A finite lattice $L$ is order-polynomially complete if and only if $L$ is simple and has no central relation.

This result is connected to the theory of clones because it states the following: Let $(A ; \leqslant)$ be a lattice ordered finite set. The maximal subclones containing the functions $\wedge$ and $\vee$ of the clone of all order-preserving functions of $A$ are either preserving a non trivial equivalence relation or a central relation.

Furthermore from the above theorem and from [10] we have the following

THEOREM 6. A simple modular lattice $L$ of finite length is a projective geometry if and only if $L$ has no central relation.

M. Szymańska has proved in [11] that a lattice $L$ of finite length where 1 is the join of atoms has no central relation. Together with Theorem 5 this gives a new proof of Satz 5 in Wille [12].

\section{References}

[1] H. Bandelt, 'Tolerance relations on lattices', Bull. Austral. Math. Soc. 23 (1981), 367-381.

[2] G. Birkhoff, Lattice theory, 3rd ed. (Amer. Math. Soc. Colloq. Publ.,25, Providence, R.I., 1967).

[3] I. Chajda, 'Recent results and trends in tolerance on algebras and varieties', Colloquia Mathematica Societatis János Bolyai 28 Szeged (1979), 69-95.

[4] J. Hashimoto, 'Congruence relations and congruence classes in lattices', Osaka J. Math. 15 (1963), $71-86$. 
[5] G. Grätzer, Universal algebra (New York, 1979).

[6] G. Grätzer and E. T. Schmidt, 'On congruence lattices of lattices', Acta Math. Acad. Sci. Hungar. 13 (1962), 178-185.

[7] M. Kindermann, 'Uber die Äquivalenz von Ordnungspolynomivollständigkeit und Toleranzeinfachheit endlicher Verbände', Contribution to general algebra, H. Kautschitsch et al., pp. 145-149 (Klagenfurt, 1979).

[8] I. G. Rosenberg, 'Uber die funktionale Vollständigkeit in den mehrwertigen Logiken', Rozpravy Československe Akad. Věd. Řada Mat. Př̈rod Věd. 804 (1970), 1-93.

[9] D. Schweigert, 'Uber endliche, ordnungspolynomvollständige Verbände', Monatsh. Math. 78 (1974), 68-76.

[10] D. Schweigert, 'Compatible relations of modular and orthomodular lattices; Proc. Amer. Math. Soc. 81 (1981), 462-463.

[11] M. Szymańska, 'On central relations of complete lattices', Preprint, Warszawa (1981).

[12] R. Wille, 'Eine Charakterisierung endlicher, ordnungspolynomvollständiger Verbände', Arch. Math. 28 (1977), 577-560.

FB Matematik

Universität Kaiserslautern

D 6750 Kaiserslautern

West Germany 